\title{
Fracture and Lateral Dislocation of the T12-L1 Vertebrae Without Neurological Deficit
}

\author{
-Case Report- \\ Kamil Melih AKaY, Alper BAySEFer, Hakan KAYAli, Altay BEDUK, \\ and Erdener TIMURKAYNAK
}

Department of Neurosurgery, Gulhane Military Medical Academy, Ankara, Turkey

\begin{abstract}
A 21-year-old man suffered T12-L1 vertebrae fracture and lateral dislocation without neurological deficit. Computed tomography and magnetic resonance imaging demonstrated the fracture and lateral dislocation of the thoracolumbar spine. The injured spine was realigned with rods and screws, and bony fusion of the affected vertebrae was performed. Patients with thoracolumbar fracture-dislocation without neurological deficit may suffer unintended neurological injury secondary to maneuvers that cause further dislocation of the spine. Severe spinal injury without neurological deficit should be evaluated in detail, especially with spinal computed tomography. Internal fixation and reduction are recommended if the patient's condition is suitable for surgery.
\end{abstract}

Key words: fracture, dislocation, etiology, diagnosis, surgery

\section{Introduction}

Severe fracture-dislocations of the thoracolumbar spine are usually associated with complete neurological dysfunction below the level of injury, but a few cases of thoracolumbar fracture-dislocation present without neurological injury. ${ }^{1-4,7,8,10,12,14)}$ The mechanism of the spine injury is probably responsible for the absence of neurological injury in these cases.

We treated a patient with T12-L1 vertebrae fracture-lateral dislocation injury without neurological injury and discuss the clinical and radiological features, mechanism of the fracture, and the management of the lesion.

\section{Case Report}

A 21-year-old male patient was admitted to our hospital following a traffic accident in which he was traveling in the rear seat without a safety belt in a car and was found outside the car after the accident. He complained of low back pain. Neurological examination found no abnormalities. No other system injury was detected. Spine radiography showed a fracture and lateral dislocation of the T-12 to L-1

Received September 30, 2002; Accepted January 23, 2003 vertebrae (Fig. 1). Emergent spinal computed tomography (CT) and magnetic resonance imaging showed various pathologies at the involved levels (Figs. 2 and 3). The right inferior facet of the T-12 vertebra, and left superior facet and pedicle of the L-1 vertebra were fractured. The coronal alignment of the spinal column at the involved levels was lost and the spinal cord appeared to be transected

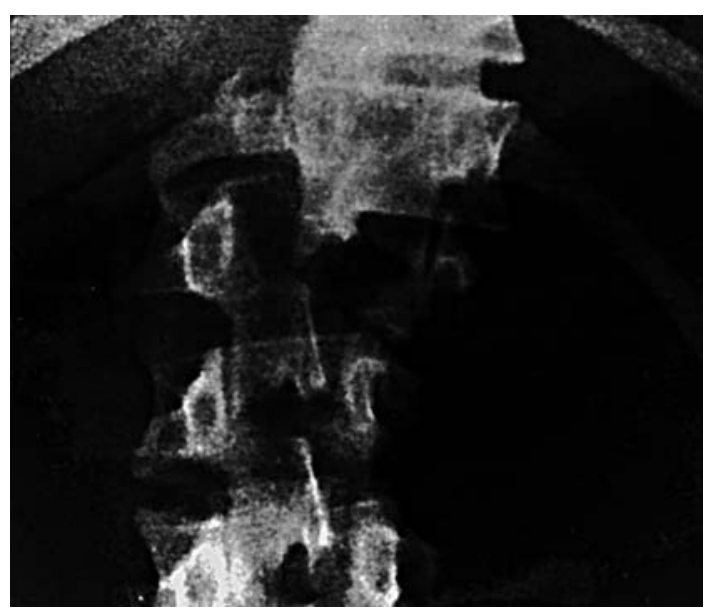

Fig. 1 Posteroanterior radiograph showing fracture and lateral dislocation of the T-12 to L-1 vertebrae. 


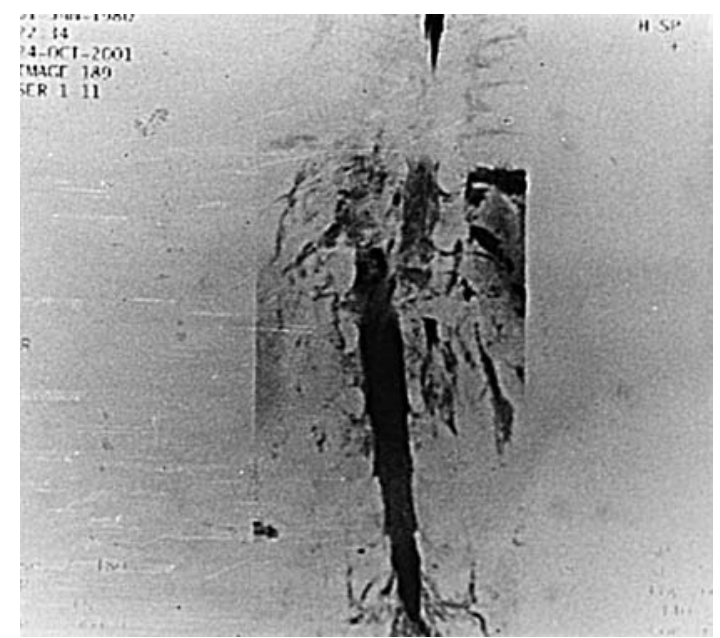

Fig. 2 Magnetic resonance myelogram showing absence of continuity of the spinal theca at the involved level.

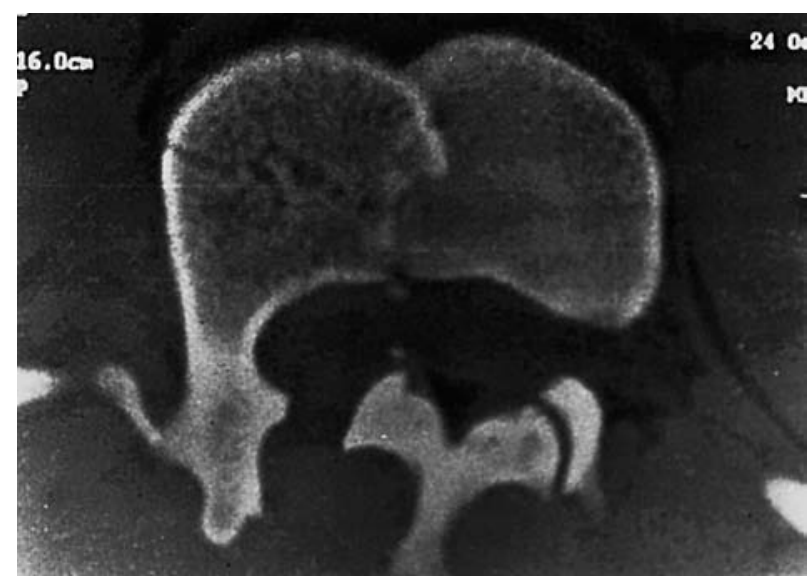

Fig. 3 Computed tomography scan of the lesion using a bone window showing the fractured left L-1 vertebra pedicle and spinal canal sparing.

(Fig. 2), despite the normal neurological function below those levels. The spine was assessed as unstable and an emergent operation was planned.

Under the general anesthesia, the patient was positioned in the prone position. There was no skin stigma. A traumatic hematoma was evacuated on the fascia following a thoracolumbar midline vertical incision. The fascia was opened in the midline and the paravertebral muscles were dissected laterally. The left pedicle of the L-1 vertebra was found to be fractured. Laminectomy and pediclectomy of the fractured vertebra were performed. The dura and spinal cord were found to be normal except for the
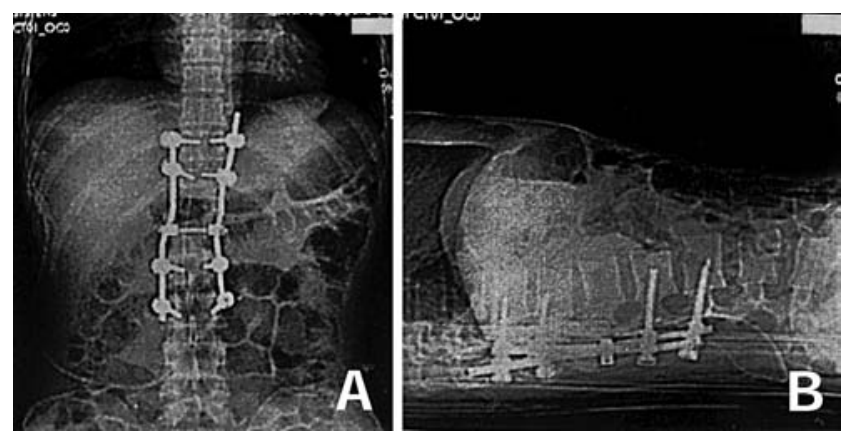

Fig. 4 Postoperative posteroanterior (A) and lateral (B) radiographs showing sagittal and coronal realignment of the fractured spine.

displacement. Then, the spinal canal was controlled with a rubber catheter and found to be open. Transpedicular screws were placed in the T-11, T-12, L-2, and L-3 vertebrae. Realignment of the injured segment of the spine was achieved with the rods (Fig. 4). Bony fusion of the transverse processes and facets of the T-12 and L-1 vertebrae was then performed. The patient was neurologically intact in the early postoperative period and was discharged at the 5 th day after surgery. He was doing well at the follow-up examination after 3 months.

\section{Discussion}

Fracture and dislocation of the thoracic or lumbar spine without neurological deficit is rare and no case involving the T12-L1 vertebrae has been reported. ${ }^{1-4,7,8,10,12,14)}$ There are two main factors that should be considered: the mechanism of the injury, and the individual bone and ligament structure of the patient.

Traffic accidents are the most common etiological factors in reported cases of thoracolumbar fracture and dislocations without neurological deficit. ${ }^{7,10,12)}$ In our case, a traffic accident in a car was the cause of the lesion. This type of fracture and dislocation seems to be caused by a powerful force.

All patients with a thoracic or lumbar fracture and dislocation without neurological deficit but with spinal cord sparing had fractures in the middle column, or the pedicles or facets of the involved vertebrae.1,10,12,13,15) Spinal cord sparing may result from bilateral pedicular fractures at the involved levels which allow the posterior elements to remain in continuity whereas the vertebral bodies become severely displaced, so spinal cord remains in its correct relationship to the posterior elements and therefore is not compromised. ${ }^{12)}$ The posterior elements 
of the involved vertebrae are broken into separate pieces and displaced posteriorly or laterally, which leads to spontaneous decompression of the dura and marked widening of the spinal canal. ${ }^{1)}$ In our case, left superior pedicular fracture of the L-1 allowed the posterior elements of the involved vertebrae to remain in continuity, which resulted in spontaneous decompression of the dura and marked widening of the spinal canal at the involved levels. Therefore, isolated fractures of the middle column elements may be considered as good prognostic features in cases of combined thoracolumbar fracture and dislocation.

The ligamentous structure is another important prognostic factor for these types of fractures. Ligamentous elasticity has been considered responsible for stability of the spinal column following spinal injuries in children. ${ }^{16)}$ Similarly, the rib cage may act as a supportive structure to help maintain the stability of the injured thoracic spine. ${ }^{12,15)}$ In our case, the ligamentous instability and probable absence of the rib cage at the involved level yielded to the excessive degree of lateral dislocation.

Diagnosis of the lesion before any dangerous maneuver to the spine is very important in patients with spinal fracture-dislocation without neurological deficit. The spinal cord is extremely vulnerable due to the relatively unstable spine, so any maneuver before diagnosis may cause unintended neurological deficits. A few cases could not be identified at first admission. ${ }^{712)}$ Therefore, all patients with severe injury (particularly after traffic accidents) should be evaluated carefully before any risky maneuver of the spine. Spinal CT with thin sections is the method of choice for assessing the lesion and stability in thoracolumbar fracture-dislocations. ${ }^{5,12)}$ Other intraabdominal organ injury may also be present. ${ }^{10,12,13)}$

Management of thoracolumbar fracture-dislocations depends upon several factors. The first choice is between conservative and surgical treatment. ${ }^{12)}$ Conservative treatment should be adopted as the first choice in older patients and in those with underlying diseases or serious complications. ${ }^{10)}$ The current management principle for thoracolumbar fracture-dislocations is reduction and internal fixation of the injured spine, which allows early mobilization of all patients regardless of neurological deficit, and protects the neural structures from further injury and enhances recovery. ${ }^{6)}$ Furthermore, surgical stabilization reduces the hospital stay, improves spinal alignment, shortens rehabilitation, and results in fewer medical complications. ${ }^{11)}$ Therefore, we preferred to carry out internal fixation and reduction in the management of our patient. Our result was good in terms of early mobili- zation and protecting the spinal cord from further injury.

Long instrumentation and short bony fusion are recommended for surgical stabilization. ${ }^{7,16)}$ Instrumentation may fail to stabilize the injured spine due to complete disruption of all of the ligaments of the spine. ${ }^{1)}$ For this reason, we performed bony fusion between the transverse processes and pedicles of the T-12 and L-1 vertebrae. The result of this fusion should be assessed after longer periods and the patient is still under radiological follow up. Intraoperative wake-up and other monitoring techniques have been proposed to prevent unintended neurological injury due to surgical correction of spinal deformities. ${ }^{7,9}$ In our case, we did not use any kind of monitoring techniques.

Thoracolumbar fracture and dislocation without neurological deficit is a rare and treatable clinical entity. Patients with thoracolumbar fracture-dislocation without neurological deficit may suffer unintended neurological injury secondary to maneuvers that may cause further dislocation of the spine. Therefore, such patients with suspected severe spinal injury without neurological deficit should be evaluated in detail, especially with spinal CT. The optimum treatment for thoracolumbar fracturedislocation lesions is internal fixation and reduction if the patient's condition is suitable for surgery. Long instrumentation and short fusion are preferred and intraoperative monitoring techniques, if available, may be utilized during the surgery.

\section{References}

1) Abdel-Fattah H, Rizk AH: Complete fracture-dislocation of the lower lumbar spine with spontaneous neurologic decompression. Clin Orthop 251: 140-143, 1990

2) Chen WC: Complete fracture-dislocation of the lumbar spine without paraplegia. Int Orthop 23: 355-357, 1999

3) de Lucas JC, Alvarez L, Abril JC, Calvo E: Fracturedislocation of the thoracic spine without neurological lesion. Injury 25: 105-107, 1994

4) Denis F, Burkus JK: Lateral distraction injuries to the thoracic and lumbar spine. A report of three cases. J Bone Joint Surg Am 73: 1049-1053, 1991

5) Gellad FE, Levine AM, Joslyn JN, Edwards CC, Bosse M: Pure thoracolumbar facet dislocation: clinical features and CT appearance. Radiology 161: 505-508, 1986

6) Jacobs RR, Casey MP: Surgical management of thoracolumbar spinal injuries. General principles and controversial considerations. Clin Orthop 189: 22-35, 1984

7) Korovessis P, Sidiropoulos P, Dimas A: Complete 
fracture-dislocation of the thoracic spine without neurologic deficit: case report. J Trauma 36: 122-124, 1994

8) Liljenquist U, Halm H, Castro WH, Mommsen U: Thoracic fracture-dislocations without spinal cord injury: a case report and literature review. Eur Spine J 4: 252-256, 1995

9) Meyer PR Jr, Cotler HB, Gireesan GT: Operative neurological complications resulting from thoracic and lumbar spine internal fixation. Clin Orthop 237: 125-131, 1988

10) Miyasaka Y, Satomi K, Sugihara S, Tahara $Y$, Hayashi T, Ishii Y: Posterior fracture-dislocation of the thoracic spine without neurologic deficit. A case report and short literature review. Spine 18: 2351-2354, 1993

11) Shaffrey CI, Shaffrey ME, Whitehill R, Nockels RP: Surgical treatment of thoracolumbar fractures. Neurosurg Clin N Am 8: 519-540, 1997

12) Simpson AH, Williamson DM, Golding SJ, Houghton GR: Thoracic spine translocation without cord injury. J Bone Joint Surg Br 72: 80-83, 1990
13) Suomalainen O, Paakkonen M: Fracture dislocation of the lumbar spine without paraplegia. A case report. Acta Orthop Scand 55: 466-468, 1984

14) Villanueva C, Pellise F, Bago J, Cardona X: Lateral distraction injury to the lumbar spine. Report of spontaneous reduction. Spine 19: 846-848, 1994

15) Voronovich IR, Dulub OI, Nikolayev VN, Selkov JI: A variant of severe deformation of the thoracic spine with favorable neurologic outcome. Spine 15: 833-834, 1990

16) Yazici M, Alanay A, Aksoy MC, Acaroglu E, Surat A: Traumatic L1-L2 dislocation without fracture in a 6year-old girl. Incomplete neurologic deficit and total recovery. Spine 24: 1483-1486, 1999

Address reprint requests to: K. M. Akay, M.D., Mercimek Sokak 10/3, Etlik, Ankara, 06010, Turkey. e-mail: kmakay45@hotmail.com. 